\section{SM Journal of Depression Research and Treatment}

\author{
Article Information \\ Received date: Feb 10, 2017 \\ Accepted date: Apr 25, 2017 \\ Published date: Apr 28, 2017 \\ *Corresponding author \\ Oscar Castañeda Sánchez, Medical unit \\ of High Epeciality Puebla, Social Security \\ Mexican Institute, 2nd street 2004, \\ Colony Center, Puebla, Puebla, 72000 , \\ México; Tel: +0122 2232 9029; Email: \\ oscarcasanchez@gmail.com
}

Distributed under Creative Commons CC-BY 4.0

Keywords Depression; Diabetes Mellitus; Risk

Article DOI 10.36876/smjdrt.1015

\section{OPEN ACCESS}

\section{Risk of Depression in Patients Carried Out of Diabetes Mellitus Attending the Diabetimss Program}

\author{
Oscar Castañeda Sánchez ${ }^{1 \star}$ and Erika Janeth Robles ${ }^{2}$ \\ ${ }^{1}$ Head of Quality Division, Social Security Mexican Institute, Mexico \\ ${ }^{2}$ Intern of nursing, Social Security Mexican Institute, Mexico
}

\section{Abstract}

Diabetes Mellitus (DM) has been associated with a variety of neuropsychiatric disorders. People with diabetes are twice as likely to suffer from depression.

Objective: To determine the risk of depression in patients with diabetes mellitus who attend the Diabetimss program.

Material and methods: A descriptive cross-sectional study was carried out in the module of Diabetimss of UMF 1 of Obregon, in June 2012. Non-probabilistic patients with diabetes mellitus were included, applying the scale of Yesavage. The results were analyzed with frequencies and proportions.

Results: Surveys were applied to 34 patients with diabetes mellitus, 27 women and 7 men with an average age of $36.9+/-13.9$ years. $11.8 \%$ had a risk of depression and $14.7 \%$ had depression.

Conclusions: According to the results of the survey, 1 in 10 patients with diabetes mellitus are at risk for depression and 1 and a half are depressed.

\section{Introduction}

Diabetes Mellitus (DM) has been associated with a variety of neuropsychiatric disorders, including major depressive disorder, schizophrenia, Parkinson's disease, mild cognitive impairment, and Alzheimer's disease. Several clinical-epidemiological studies, and even neuroimaging, support the association between these neuropsychiatric entities and DM. Case-control studies, as well as cohort studies, have documented the increased prevalence and incidence of depression in DM. Relating the presence of depression associated with diabetes with poor metabolic control, increased complications and even increased mortality [1].

In diabetic patients with a certain frequency, there is depression of different severity, both in the period of mourning that accompanies the knowledge of the diagnosis, and by the changes in habits that implies the management of the disease. Thus, people with diabetes are twice as likely to suffer from depression; however, it is up to each person that diabetes precedes or happens the onset of depression [2].

It is estimated that the prevalence of depression in the general population is $6 \%$. In diabetic patients, according to some references, this percentage rises to $18 \%$. Various psychological difficulties, such as dietary restrictions, increased costs associated with therapeutics and frequent hospitalizations, accompany DM and may contribute to the onset of depression. Other phenomena that belong to the field of psychology as alterations in cognitive function or an inability to learn have been gradually collected from popular psychological theories and then, in most cases, empirically hypothesized to explain the development of depression in Patients with DM [3].

Lustman reports that the person with diabetes can develop practically any psychiatric syndrome, with the most common disorders being anxiety and depression, however, the magnitude of the problem is poorly understood in patients of primary care in terms of prevalence and complexity. Only $37 \%$ are detected, being confused the association with sociodemographic and clinicometabolic variables, mainly glycosylated hemoglobin (HbA1c) and control indicators, as well as patterns that lead to evolutionary stages where depression is more frequently present, either to prevent it, to decrease or to delay complications [4].

Different studies have shown that in type $1 \mathrm{DM}$, the prevalence of depression among the young population is not higher than in the general population. However, the likelihood of depressive status increases as glycemic control worsens and, at the same time, when a depressive disorder appears in a person with $\mathrm{DM}$, glycemic control worsens. In type $2 \mathrm{DM}$, when it is not associated with other chronic diseases or with specific complications of DM, it seems not to be proven to increase the risk of depression. Patients with type $2 \mathrm{DM}$ and depression usually have low adherence to treatment and 


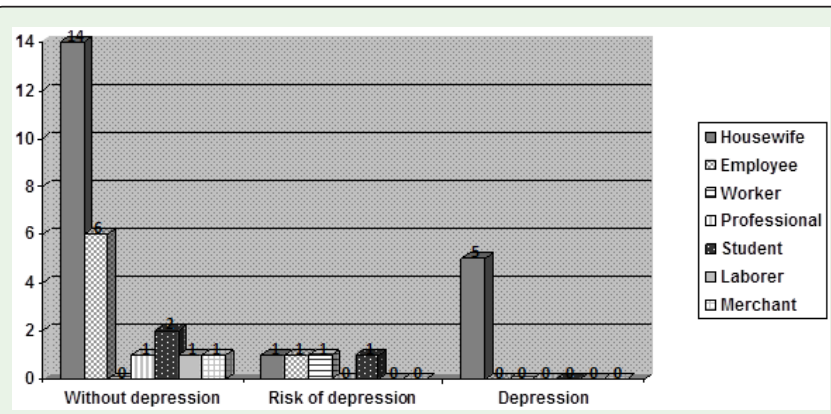

Figure 1: Changes in Mean Facial ( \pm S.E.) Width-to-Height Ratios of Major League Baseball Players Active 1870-1989.

it seems that depression in this type of patients is a predictive variable of therapeutic compliance [5].

When comparing groups of diabetics with disease-free subjects, it has been found that the risk of depression is almost twice as high among the former, with no clear differences according to gender and type of DM [6].

Depression interacts with diabetes in more than one mechanism. It can be a detonating factor of hyperglycemia, a therapeutic barrier or a consequence of the impact of the disease [7]. Thus, diabetes has a great psychological impact, as evidenced by numerous studies that report an increase in psychiatric morbidity among those who suffer from it. Being a common psychiatric diagnosis in the patients with this disease [8]. Therefore, this study aimed to determine the risk of depression in patients with diabetes mellitus who attend the Diabetimss program.

\section{Material and Methods}

A descriptive cross - sectional study was performed in the Diabetimss module of the Family Medicine Unit No. 1 of the IMSS of Ciudad Obregon, Sonora, medical unit of first level care. Patients with diabetes mellitus who attended the diabetes module were included in a non-probabilistic manner. The Yesavage scale was applied to participants, thinking that the majority of the patients are older adults. The instrument is already validated and consists of 30 questions with which the risk of presenting depression is determined, responding to each reagent whether or not, rating their results as follows: 0 to 10 positive responses $=$ no depression, 11 to 14 positive responses $=$ risk of depression and more than 14 positive responses $=$ presence of depression.

The variables of the study were: age, sex, occupation, schooling, score and presence of risk of depression. Analyzing the results for

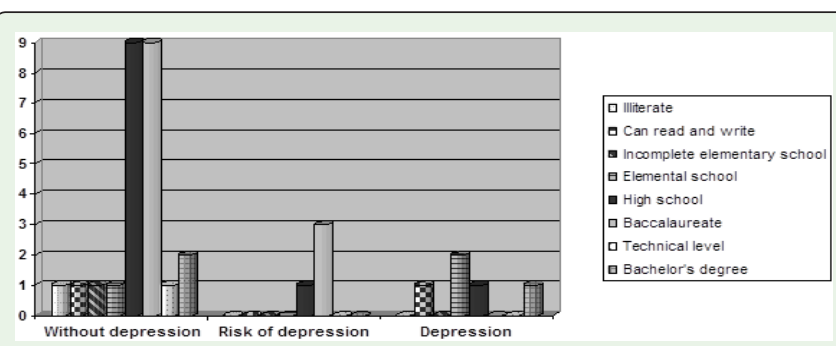

Figure 2: Distribution of the risk of depression according to their level of schooling.

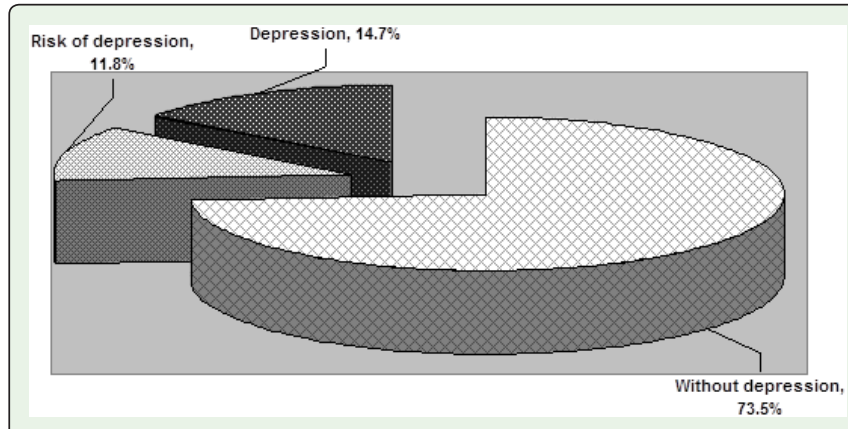

Figure 3: Risk of depression in patients with Diabetes Mellitus who attend to Diabetimss.

the case of the qualitative variables with frequencies and proportions. And in the case of age and score were applied measures of central tendency.

\section{Results}

We interviewed 27 (79.4\%) women and 7 (20.6\%) men, with an average age of $36.9+/-13.3$ years. Of these, most of their occupation is housewives as shown in (Figure 1). Meanwhile, their level of schooling is predominantly high school as shown in (Figure 2).

The results of the interview showed scores ranging from 0 to 22 , with an average of $7.6+/-5.8$. According to which, the presence of depression occurred in five cases as shown in (Figure 3).

\section{Discussion and Conclusion}

The results after the evaluation of the applied surveys show that a quarter of the population studied is at risk or has depression. However, these results are lower than those reported by Colunga et al (2008), where the probability of the condition was found in $63 \%$ of the population studied. Showing differences in the level of schooling, since, in that study was smaller, although the occupation was very similar (49\% housewives). And with regard to sex, as in this study, women were the most affected.

Another study by Escobedo Lugo et al, carried out in a sample of similar size, showed a higher risk of depression (82.60\%), and an average age greater than that of this work $(51+/-11$ years).

The results of Dorta et al (2014), coincide with those of this study, by showing the risk factors that play a role in the presence of depression in people with diabetes mellitus, occupation as housewife and elemental schooling [9].

In conclusion, according to the results of the surveys applied to patients with diabetes mellitus, 1 in 10 patients presented a risk for the development of depression and in 1 and a half the presence of depression was demonstrated. With regard to occupation, the risk of depression was predominantly presented in housewives. And in relation to their educational level, the risk of depression predominates in those with high school education and the presence of depression in those with elemental schooling.

\section{References}

1. Castillo-Quan JI, Barrera-Buenfil DJ, Pérez-Osorio JM, Álvarez-Cervera FJ. Depression and diabetes: from epidemiology to neurobiology. Rev Neurol. 2010; 51: 347-359. 
2. Pineda N, Bermúdez V, Cano C, Mengual E, Romero J, Medina M et al. Levels of depression and symptomatology characteristic in adult patients with type 2 diabetes mellitus. AVFT. 2004; 23: 74-78.

3. Benítez A, Gonzáles L, Bueno E, Agüero F, Alsina S, Melgarejo M et al. Depression and diabetes. Rev ALAD. 2011; 1: 162-169.

4. Colunga-Rodríguez C, García de Alba JE, Salazar-Estrada JG, AngelGonzález M. Type 2 Diabetes and Depression in Guadalajara, Mexico. 2005. Rev Salud Publica. 2008; 10: 137-149.

5. Peralta-Pérez G. Depression and diabetes. Av Diabetol. 2007; 23: 105-108.

6. Maldonado JC. Diabetes mellitus y depresión. Rev Fac Cienc Med (Quito). 2009; 34: 3-4.
7. Castillo-Nuñez Y, Aguilar-Salinas CA. Depression, glycemic control and diabetes. Rev Alad. 2011; 1: 142.

8. Escobedo-Lugo C, Díaz-García NA, Lozano-Nuevo JJ, Rubio-Guerra AF Varela Gutiérrez G. Metabolic uncontrol related to depression according to the inventory of Beck in diabetic patients type 2 of recent diagnosis. Med Int Mex. 2007; 23: 385-390.

9. Dorta L, Martes M, Villalba $P$, Fuentes $P$, Pérez Peñaranda A. Risk of depression in patients with type 2 diabetes mellitus according to the time of evolution. Diabetológico Center Aragua, Venezuela, 2011. Comunidad y Salud. 2014; 12: 33-38. 